\title{
Reducing Door to- Balloon- Time for Acute ST Elevation Myocardial Infarction In Primary Percutaneous Intervention: Transformation using Robust Performance Improvement
}

\author{
Samer Ellahham, MD, Samir Aljabbari, Tristan Harold Mananghaya, Salama J. Raji, Abdulmajeed Al Zubaidi \\ SKMC
}

\begin{abstract}
Cardiovascular diseases (CVDs) are the leading causes of death in the UAE. Prompt reperfusion access is essential for patients who have Myocardial Infarction (MI) with ST-segment elevation as they are at a relatively high risk of death. This risk may be reduced by primary percutaneous coronary intervention (PCl), but only if it is performed in a timely manner. Guidelines recommend that the interval between arrival at the hospital and intracoronary balloon inflation (door-to-balloon (D2B) time) during primary PCl should be 90 minutes or less. The earlier therapy is initiated, the better the outcome. Our aim was to decrease the door-to-balloon time for patients with ST segment elevation myocardial infarction (STEMI) who come through the emergency department (ED) in Sheikh Khalifa Medical City, a tertiary hospital in UAE, to meet the standard of less than 90 minutes. A multidisciplinary team was formed including interventional cardiologists, catheterization laboratory personnel, emergency department caregivers and quality staff. The project utilized the Lean Six Sigma Methodology which provided a powerful approach to quality improvement. The process minimized waste and variation, and a decreased median door-to-balloon time from 75.9 minutes to 60.1 minutes was noted. The percentage of patients who underwent PCI within 90 minutes increased from $73 \%$ to $96 \%$. In conclusion, implementing the Lean Six Sigma methodology resulted in having processes that are leaner, more efficient and minimally variable. While recent publication failed to provide evidence of better outcome, the lessons learned were extrapolated to other primary percutaneous coronary intervention centers in our system. This would have marked impact on patient safety, quality of care and patient experience.
\end{abstract}

\section{Problem}

SKMC is the primary center which performs $\mathrm{PCl}$ as treatment for Myocardial Infarction (MI) in Abu Dhabi, UAE. Timely access and intervention are life saving and have the potential of improving outcome. Primary $\mathrm{PCl}$ is the routine treatment in SKMC for eligible STEMI patients 24 hours a day, 7 days a week. Guidelines recommend that the interval between arrival at the hospital and intracoronary balloon inflation (door-to-balloon (D2B) time) during primary $\mathrm{PCl}$ should be 90 minutes or less(1-5).

The primary challenge addressed by the SKMC STEMI protocol was the suboptimal coordination of care for patients with STEMI from the emergency department (ED) to the cardiac catheterization laboratory. Although each group of care givers strived to optimize processes within their own work unit, we felt however that more coordinated effort was needed to improve timeliness of primary PCI across the entire system of care experienced by the patient.

\section{Background}

Rapid reperfusion in patients with ST-elevation myocardial infarction (STEMI) is associated with better outcome. Reduction in door-to-balloon (D2B) time in primary $\mathrm{PCl}$ requires multidisciplinary cooperation, process analysis and quality improvement methodology.Implemententation of an innovative methodology for improving health care services is indicated.

Lean and Six Sigma are strategies to improve process efficiency and quality. During the past decade, these process improvement techniques have been successfully applied in health care. Lean involves a set of principles, practices and methods for designing, improving and managing processes. The development of Lean is attributed to Taiichi Ohno's articulation of the Toyota Production System. Ohno aimed to improve efficiency by eliminating particular kinds of waste (called muda, in Japanese) which absorb time and resources but do not add value.Six Sigma, like Lean, is a business management strategy used to improve the quality and efficiency of operational processes. While Lean focuses on identifying ways to streamline processes and reduce waste, Six Sigma aims predominantly to make processes more uniform and precise through the application of statistical methods. The Six Sigma improvement model, Define, Measure, Analyze, Improve, and Control (DMAIC) specifies the following sequence of steps for understanding and improving a process: 1 ) defining the project goals and customer (internal and external) requirements; 2) measuring the process to determine current performance; 3 ) analyzing and determining the root cause(s) of relevant defects; 4) improving the process by eliminating defect root causes, and 5) controlling future process performance. (6-15)

\section{Baseline measurement}

For three consecutive months, from September to November 2011, only $73 \%$ of our STEMI patients had D2B times less than 90 minutes. In order to increase this rate, our average D2B time should be reduced. To achieve this goal, the first objective was to identify the steps (metric) from medical contact (door) to open artery, along 


\section{BMJ Quality Improvement Reports}

with the observed time for each step in the process. The main categories of process steps include the door-to-ECG, ED to cardiac catheterization laboratory, and catheterization laboratory time. D2B is a time measurement in emergency cardiac care (ECC), specifically in the treatment of ST segment elevation myocardial infarction (or STEMI). The interval starts with the patient's arrival in the emergency department, and ends when a catheter guidewire crosses the culprit lesion in the cardiac cath lab. The primary outcome measure was overall door-to-balloon time.Subinterval time periods were collected to show what effect the changes had on the sub-processes in the overall door-to-balloon process.

See supplementary file: ds5421.jpg - "Baseline Measurement"

\section{Design}

A multidisciplinary meeting involving concerned stakeholders was called in order to determine the project's viability as a Six Sigma project, prior to the creation of the project charter which authorized, formalized and assigned responsibilities within the project. A viability matrix was formed and the total score based on the agreed matrix is 4.16. Having a score greater than 3 meant that the process would lend itself well to Lean Six Sigma. The choice of Lean Six Sigma methodology was based on the remarkable wastes noted, limited resources and the significant variation of the process.

\section{Strategy}

Lean Six Sigma methodology was used to reduce D2B times in STEMI patients presenting to our tertiary care center. Specific steps in STEMI care were determined, time goals were established, and processes were changed to reduce each step's duration. Outcomes were tracked, and timely feedback was given to providers.

Several strategies implemented at each door-to-balloon interval in our protocol have contributed to the ability to obtain door-to-balloon times of $\leq 90$ minutes in such a large proportion of patients with STEMI. Changes in flow of care, communications, cardiac catheterization team activation and data collection were made. Changes in practice implemented at SKMC were based on prior published suggestions on how to decrease door-to-balloon. Other changes were made based on the DMAIC cycle performed through this project. The multidisciplinary team analyzed each phase in our door-to-balloon process using the data collected, then applied current process reviews, lean thinking and brainstorming. The team came up with the fishbone diagram which clearly identifies the causes of the delay in our door and value stream mapping was performed.

Details of the DMAIC methodology adopted:

Define:

Project Goal

Improve access and processes that will lead to meeting the recommended door-to- balloon time of 90 minutes
Project Scope

Start : Patient arrival at SKMC

Stop : Establishment of normal blood flow

Includes : STEMI, Primary PCI

Excludes : All other cases

Measure:

We measured the process by taking note of the time required to finish each phase of the D2B process.

Analyze:

We used process mappping and fishbone diagram to identify causes of wastes, and analyzed our data further using control charts, histograms, boxplots and pareto charts.

Improve:

Redesigned the $\mathrm{D} 2 \mathrm{~B}$ process and prevented delay by adhering to the key processes.

Direct activation of the Cathlab by the ED Senior Specialist or Consultant.

Effective scheduling of the CathLab team to ensure availability during their activation.

Standardized the time documentation by consistently using Cerner time.

Reformatted the D2B time entry form to ensure correct documentation- made it lean and easy to follow.

Control:

Tracking, auditing and monitoring the $\mathrm{PCl}$ performance improvement opportunities.

Availability of an ED Senior Specialist or Consultant at all times.

Conducted monthly multidisciplinary meetings to evaluate the $\mathrm{PCl}$ process effectiveness. Ensured timely feedback with the involved departments.

Correct staff documentation.

Using a single-call page system.

Effective shift scheduling and avoidance of staff shortage.

\section{Results}

With our D2B process significantly improved, our median D2B times 
during the months of February to April 2012 decreased from 75.9 to 60.1 minutes. In addition, the percentage of patients meeting the 90-minute window improved from $73 \%$ to $96 \%$. Improvements from there on were sustained and targets were achieved. Strong and optimal interdepartmental communication were also observed since the role of the different departments in each of the care process were determined, clarified and agreed upon. It also ensured that prompt feedback of time-based results to staff involved in the care of the STEMI patients were shared and discussed.

See supplementary file: ds5422.jpg - "Before and After Processes and Results"

\section{Lessons and limitations}

The focus in the project was reduction in time. The first step in both projects was to secure reliable and valid measurements. The project team used time as the best overall measure. By using the measurements in each and every step in both projects and adding it to value stream mapping, the team excelled trough brain storming and deep analyses.

SKMC practice changes were implemented. Data was carefully collected, it was then evaluated by the multidisciplinary team leadership for accuracy and quality control. Physician support has been instrumental in making these changes happen. Improvement processes and results have been readily shared with the team involved with the STEMI patients.

Important concepts that assisted in this success were:

Get the right people involved.

A multidisciplinary team assists in identifying problems/issues, and helps to make changes easier.

Communication to all areas of this project is a necessity.

Use of the Lean and DMAIC cycle assisted us in getting rapid changes completed.

Make the process as simple as possible.

Sharing the data at real time, via graphs, charts, etc, assists others to see the progress and also areas for improvement.

Leadership and management support was paramount in setting priorities and providing directions by empowering the frontline staff who own the process.

"Culture eats strategy for breakfast", hence our hospital made a commitment early on to adopt a change management to help implement the journey of Primary angioplasty.

The essence of the project was to engage everyone in identifying and solving problems.

Using value stream mapping, a new tool recently taught to the staff helped them identify opportunities to eliminate process waste; this led to marked reduction in delay and waiting time.

\section{Limitations}

When we instituted our improved SKMC STEMI protocol, we implemented several changes simultaneously. Hence, it is impossible to differentiate which of these changes made the greatest difference in performance.

\section{Conclusion}

Continuous quality improvement for any policy or procedure at a medical facility is a continually evolving goal that is, by design, never reached. Primary percutaneous coronary intervention (PCI) IS is an effective treatment strategy for acute STsegment-elevation myocardial infarction (STEMI). Current guidelines for the treatment of ST-segment elevation myocardial infarction recommend a door-to-balloon time of 90 minutes or less for patients undergoing primary percutaneous coronary intervention (PCI). Door-to-balloon time has become a performance measure and is the focus of regional and national quality-improvement initiatives.By implementing the Lean Six Sigma methodology and multidisciplinary team biulding, SKMC STEMI protocol implemented strategies to reduce D2B for patients with STEMI. D2B was significantly reduced, and the results were sustained. Our experience demonstrates the effectiveness and durability of process changes targeting timeliness of primary percutaneous coronary intervention. we were able to achieve overall significant decrease in the average door-to-balloon time (D2B),enhanced patient care, alignment with SKMC Priorities,improved access to care and patient safety.

The processes became leaner, more efficient and minimally variable.

Achieving the 90-minute gold standard is possible, but it will require sustained investment on the part of institutions and caregivers alike.Lean management approach serves as a foundation for innovation throughout the healthcare projects. This case study provided in this paper of a UAE hospital succeeded in Lean innovation application.

By implementing the Lean Six Sigma methodology, we were able to achieve:

Overall significant decrease in the average door-to-balloon time (D2B)

Alignment with SKMC Priorities

Enhanced patient care

Improved access to care

Patient safety

D2B KPI as required by SEHA, JCIA and SCPC Chest Pain 


\section{BMJ Quality Improvement Reports}

Accreditation and sustain the result

Leaner, more efficient and minimally variable processes

Efficient allocation of resources, which includes effective staff scheduling and value-based processes

The lessons learned may be extrapolated to other primary percutaneous coronary intervention centers. While door-to-balloon time is a key requirement for any successful primary angioplasty program, a large observational study of almost 100000 patients showing that despite improvements in D2B time, there is no change in unadjusted or adjusted mortality.

\section{References}

1. World Health Organization - Noncommunicable Diseases (NCD) Country Profiles , 2014.

2. Antman EM, Anbe DT, Armstrong PW, Bates ER, Green LA, Hand M, Hochman JS, Krumholz HM, Kushner FG, Lamas GA, Mullany CJ, Ornato JP, Pearle DL, Sloan MA, Smith SC Jr, Alpert JS, Anderson JL, Faxon DP, Fuster V, Gibbons RJ, Gregoratos G, Halperin JL, Hiratzka LF, Hunt SA, Jacobs AK. ACC/AHA guidelines for the management of patients with ST-elevation myocardial infarction: a report of the American College of Cardiology/American Heart Association Task Force on Practice Guidelines (Committee to Revise the 1999 Guidelines for the Management of Patients With Acute Myocardial Infarction). J Am Coll Cardiol. 2004; 44: E1-E211.

3. Spertus JA, Eagle KA, Krumholz HM, Mitchell KR, Normand SL. American College of Cardiology and American Heart Association methodology for the selection and creation of performance measures for quantifying the quality of cardiovascular care. Circulation. 2005; 111: 1703-1712.

4. Krumholz HM, Anderson JL, Brooks NH, Fesmire FM, Lambrew CT, Landrum MB, Weaver WD, Whyte J, Bonow RO, Bennett SJ, Burke G, Eagle KA, Linderbaum J, Masoudi FA, Normand SL, Pina IL, Radford MJ, Rumsfeld JS, Ritchie JL, Spertus JA. ACC/AHA clinical performance measures for adults with ST-elevation and non-ST-elevation myocardial infarction: a Report of the American College of Cardiology/American Heart Association Task Force on Performance Measures (Writing Committee to Develop Performance Measures on ST-Elevation and Non-STElevation Myocardial Infarction). Circulation. 2006; 113: 456-462.

5. Angeja BG, Gibson CM, Chin R, Frederick PD, Every NR, Ross AM, Stone GW, Barron HV. Predictors of door-toballoon delay in primary angioplasty. Am J Cardiol. 2002; 89: 1156-1161.

6. Ohno T. Toyota Production System: Beyond Large-Scale Production. Productivity Press; New York: 1988. Original Japanese edition published in 1978 by Diamond Press.

7. Womack J, Jones D. Lean Thinking, Banish Waste and Create Wealth in Your Corporation. Simon \& Schuster; New York: 1996. pp. 15-16.

8. Hackman JR, Wageman R. Total quality management: empirical, conceptual, and practical issues. Admin Sci Quart. 1995;40:309-342.

9. Powell A, Rushmer R, Davies H. Effective quality improvement: TQM and CQI approaches. Brit J HEALTHCARE MANAGE. 2009;15(3):114-120.

10. Bendell T. A review and comparison of Six Sigma and the Lean organizations. TQM Magazine. 2006;18(3):255-262.

11. Kwak YH, Anbari FT. Benefits, obstacles, and future of six sigma approach. Technovation. 2006;26:708-715.

12. Arnheiter ED, Maleyeff J. The integration of lean management and Six Sigma. TQM Magazine. 2005;17(1):5-18.

13. George ML. Lean Six Sigma: Combining Six Sigma Quality with Lean Speed. McGraw-Hill Professional; New York, NY: 2002.

14. Berwick DM. Continuous improvement as an ideal in health care. N Engl J Med. 1989;320(1):53-56. [PubMed]

15. Laffel $G$, Blumenthal $D$. The case for using industrial quality management science in health care organizations. JAMA. 1989;262(20):2869-2873. [PubMed]

16. Door-to-Balloon Time and Mortality among Patients Undergoing Primary PCl

Daniel S.Menees et al; N Engl J Med 2013; 369:901-909

\section{Declaration of interests}

None of the authors have any declared conflict of interest related to this publication.

\section{Acknowledgements}

Many thanks to the Door-to-Balloon team members along with the care givers from the emergency department, the cardiac catheterization laboratory and the quality department, whose passion, perseverance, and dedication are responsible for the outcomes achieved.

\section{Ethical approval}

This project was deemed exempt from ethical approval as it is an improvement study and not a study on human subjects, and hence ethical approval was not required. 